Abstract of paper for AIAA $16^{\text {th }}$ Computational Fluid Dynamics Conference Orlando, Florida, June 23-26, 2003

\title{
Calculation of Supersonic Combustion Using Implicit Schemes
}

\author{
Seokkwan Yoon \\ NASA Ames Research Center \\ Moffett Field, CA 94035
}

\section{Introduction}

One of the technology goals of NASA for advanced space transportation is to develop highly efficient propulsion systems to reduce the cost of payload for space missions. Developments of rockets for the second generation Reusable Launch Vehicle (RLV) in the past several years have been focused on low-cost versions of conventional engines. However, recent changes in the Integrated Space Transportation Program to build a crew transportation vehicle to extend the life of the Space Shuttle fleet might suggest that airbreathing rockets could reemerge as a possible propulsion system for the third generation RLV to replace the Space Shuttle after 2015. The weight of the oxygen tank exceeds thirty percent of the total weight of the Space Shuttle at launch while the payload is only one percent of the total weight. The air-breathing rocket propulsion systems, which consume oxygen in the air, offer clear advantages by making vehicles lighter and more efficient

Experience in the National Aerospace Plane Program in the late 1980s indicates that scramjet engines can achieve high specific impulse for low hypersonic vehicle speeds. Whether taking a form of Rocket Based Combined Cycle (RBCC) or Turbine Based Combined Cycle (TBCC), the scramjet is an essential mode of operation for air-breathing rockets. It is well known that fuel-air mixing and rapid combustion are of crucial importance for the success of scramjet engines since the spreading rate of the supersonic mixing layer decreases as the Mach number increases.

A factored form of the Gauss-Seidel relaxation method ${ }^{1}$ has been widely used in hypersonic flow research since its first application to non-equilibrium flows. ${ }^{2,3}$ However, difficulties in stability and convergence have been encountered when there is strong interaction between fluid motion and chemical reaction, such as multiple fuel injection problems. The present paper reports the results from investigation of the effect of modifications to the original algorithm on the performance for multiple injectors.

* Research Scientist, MS T27B-1 


\section{Num erical Method}

Let $t$ be time; $Q$ the vector of conserved variables; $E, F$, and $G$ the convective flux vectors; $E_{v}, F_{v}$, and $G_{v}$ the flux vectors for the viscous terms. The source term $S$ represents production or destruction of species due to chemical reactions. Then the threedimensional Navier-Stokes and species transport equations in generalized curvilinear coordinates $(\xi, \eta, \varsigma)$ can be written as

$$
\partial_{t} Q+\partial_{\xi}\left(E-E_{v}\right)+\partial_{r}\left(F-F_{v}\right)+\partial_{s}\left(G-G_{v}\right)=S
$$

The governing equations are integrated in time for both steady and unsteady flow calculations. For a steady-steady solution, the use of a large time step leads to a fast convergence. For a time-accurate solution, it is desirable that the time step is determined by the physics rather than the numerical stability. An unfactored implicit scheme can be obtained from a nonlinear implicit scheme by linearizing the flux vectors about the previous time step and dropping terms of second and higher orders.

$$
\left[I+\alpha \Delta t\left(D \leftleftarrows A+D_{\eta} B+D_{\subsetneq} C-H\right)\right] \delta Q=R H S
$$

where

$$
R H S=-\Delta t\left[D_{\xi}\left(E-E_{v}\right)+D_{\eta}\left(F-F_{v}\right)+D_{\zeta}\left(G-G_{v}\right)-S\right]
$$

$I$ is the identity matrix and $\delta Q$ denotes the correction. $A, B, C$, and $H$ are the Jacobian matrices of the convective flux vectors and the source term respectively. Artificial dissipation models augment finite volume formulation of the right hand side. ${ }^{4}$ Direct inversion of a large block banded matrix becomes impractical in three dimensions because of the rapid increase of computational work and the large storage requirement. The LU-SGS scheme is one of the approximate factorization methods to alleviate the difficulties in three dimensions.

$$
L D^{-1} U \delta Q=R H S
$$

where

$$
\begin{aligned}
& L=I+\alpha \Delta t\left(D_{\xi}^{-} A^{+}+D_{n}^{-} B^{+}+D_{\xi}^{-} C^{+}-A^{-}-B^{-}-C^{-}-H\right) \\
& D=I+\alpha \Delta t\left(A^{+}+B^{+}+C^{+}-A^{-}-B^{-}-C^{-}\right) \\
& U=I+\alpha \Delta t\left(D_{\xi}^{+} A^{-}+D_{\eta}^{+} B^{-}+D_{\xi}^{+} C^{-}+A^{+}+B^{+}+C^{+}\right)
\end{aligned}
$$


Recent discussions ${ }^{5}$ suggest that a different treatment of the source Jacobians might increase numerical stability. For example,

$$
\begin{aligned}
& L=I+\alpha \Delta t\left(D_{\xi}^{-} A^{+}+D_{\eta}^{-} B^{+}+D_{\zeta}^{-} C^{-}-A^{-}-B^{-}-C^{-}-\frac{1}{2} H\right) \\
& D=I+\alpha \Delta t\left(A^{+}+B^{+}+C^{+}-A^{-}-B^{-}-C^{-}-\frac{1}{2} H\right) \\
& U=I+\alpha \Delta t\left(D_{\xi}^{+} A^{-}+D_{\eta}^{\prime} B^{-}+D_{\zeta}^{+} C^{-}+A^{+}+B^{+}+C^{+}-\frac{1}{2} H\right)
\end{aligned}
$$

Here, the split Jacobian matrices are constructed approximately using spectral radii to yield diagonal dominance.

In the thermodynamic model, mass-averaged $C p$ is assumed to be a fourth order polynomial function of temperature with the coefficients obtained from curve fit data. Gas mixture's $C p$ is obtained from mass concentration weighting. Internal energy of the gas mixture is determined using mixture properties including enthalpy, which is defined using the reference enthalpy (the heat of formation of species) and the integral of $C p$ with respect to temperature. Then the temperature is found from the internal energy using Newton iteration since $C p$ is assumed to be a function of temperature only. Pressure is obtained from Dalton's law of partial pressure. Equivalent specific heat ratio (sensible enthalpy/internal energy) is used for the Jacobian matrices.

In the transport property model, viscosity and thermal conductivity for individual species are calculated using the fourth order polynomial expressions. Viscosity and conductivity for the mixture are obtained from Wilke's mixing rule. Binary mass diffusivity is modeled using Chapman-Enskog formula for dilute gases in conjunction with LennardJones intermolecular potential energy functions. The inter-collision parameter, the effective collision integral factor, and the effective temperature are approximated. The diffusion velocity of each species is calculated using Fick's law and the approximated coefficients for binary diffusion between each species and the mixture.

In the finite-rate-chemistry model, the rate of change of species concentration is defined by the movement of each species summed over all of the contributing reactions (the law of mass action). Forward and backward reaction rate coefficients are calculated from the Arrhenius form. A nine-species eighteen-step reaction model for hydrogen-air is adopted. Influence of other bodies on a particular reaction is modeled using a third body efficiency correction. 


\section{Preliminary Results}

In the first test case, hydrogen fuel is injected transversely to incoming supersonic flow of air. The incoming air speed and temperature are Mach 2 and 1,000 $\mathrm{K}$. Hydrogen is injected at the sonic speed while non-catalytic wall is cooled at $600^{\circ} \mathrm{K}$. In Figure $1 \mathrm{a}$ and $\mathrm{lb}$, temperature contours for non-reacting and reacting flows are compared. Increased pressure near the exit due to combustion in Figure $2 b$ can be compared to pressure contours for non-reacting flow in Figure $2 a$.

When the air speed is increased to Mach 4 with $1,300^{\circ} \mathrm{K}$, a lot of fuel exits without combustion due to insufficient mixing as shown in Figure 3a. It is shown in Figures $3 \mathrm{~b}$ $3 \mathrm{~d}$ that various multiple injectors might enhance combustion efficiency. The effect of different implicit schemes on the robustness and efficiency of the reacting flow solver for supersonic combustion problems will be discussed in the final manuscript.

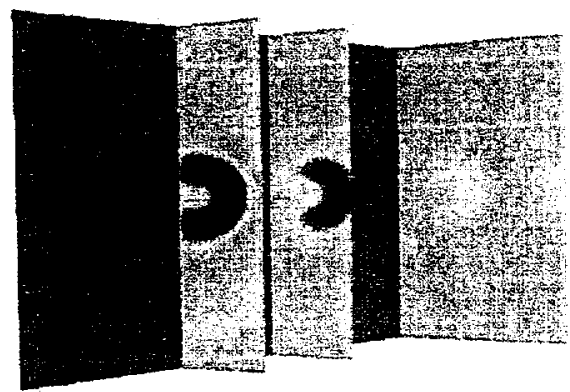

Figure 1a. Temperature for frozen flow at Mach 2

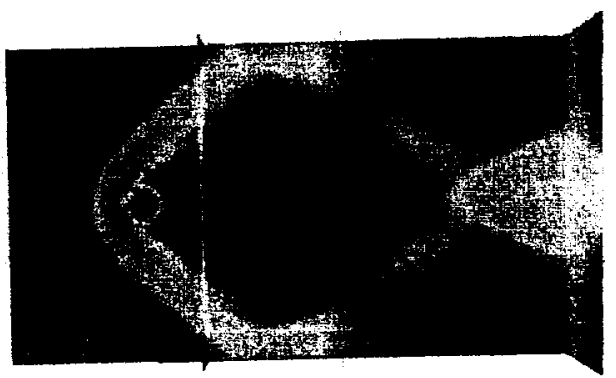

Figure 2a. Pressure for frozen flow at Mach 2

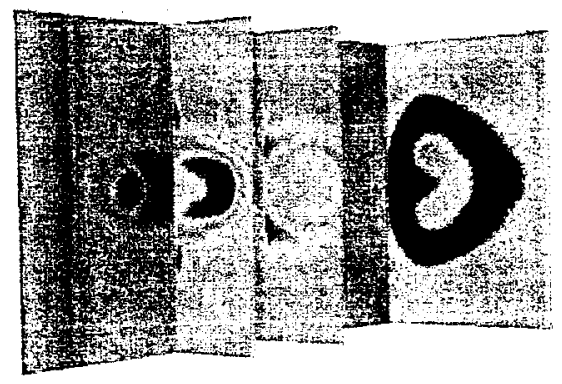

Figure 1b. Temperature for reacting flow at Mach 2

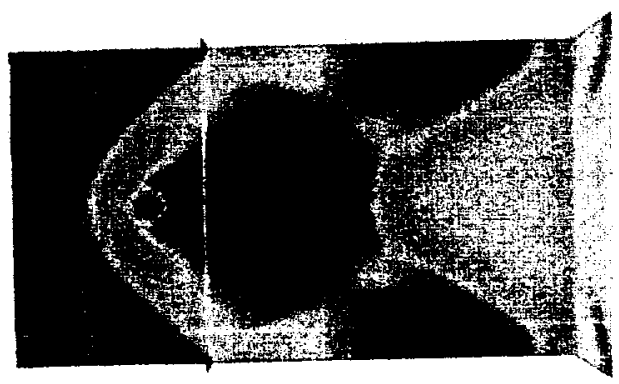

Figure $2 b$. Pressure for reacting flow at Mach 2 


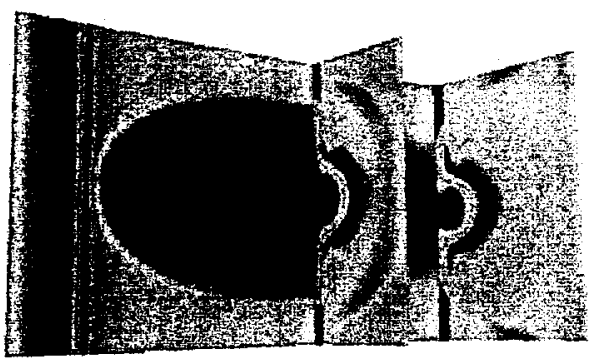

Figure 3a. Temperature for single injector at Mach 4

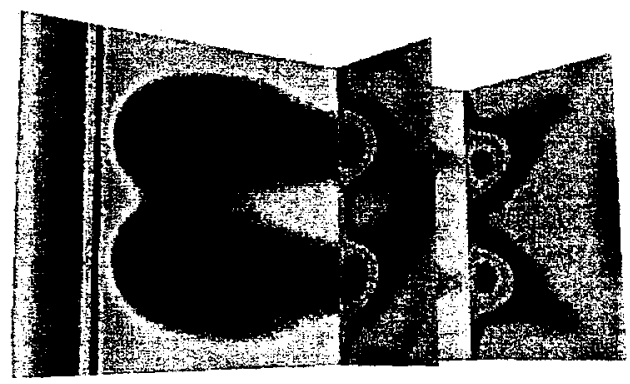

Figure 3c. Temperature for parallel injectors at Mach 4

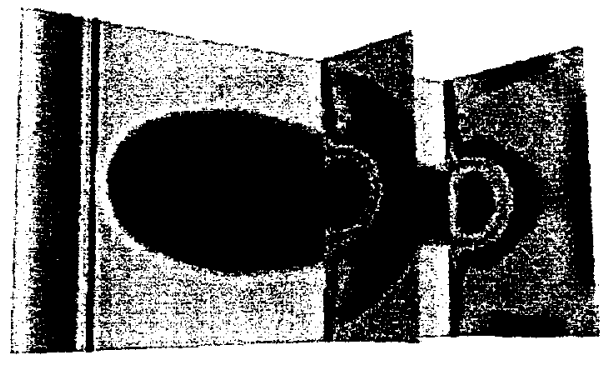

Figure $3 b$. Temperature for serial injectors at Mach 4

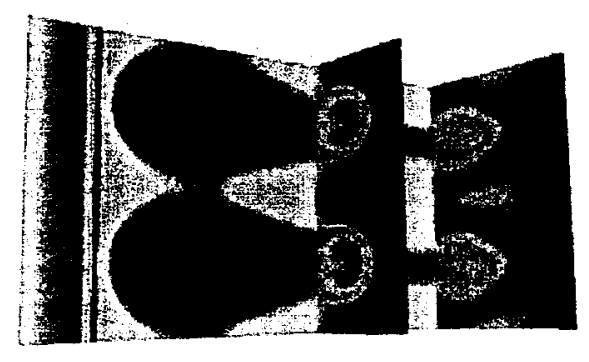

Figure 3d. Temperature for parallel-serial injectors at Mach 4

\section{References}

1. Yoon, S. and Jameson, A., "Lower-Upper Symmetric Gauss-Seidel Method for the Euler and Navier-Stokes Equations," AIAA Journal, Vol. 26, Sept. 1988, pp. 1025-1026.

2. Shuen, J.S. and Yoon, S., "Numerical Study of Chemically Reacting Flows Using an LU-SSOR Scheme," AIAA Journal, Vol. 27, Dec. 1989, pp. 1752-1760.

3. Park, C. and Yoon, S., "Fully-Coupled Implicit Method for Thermo-Chemical Nonequilibrium Air at Suborbital Flight Speeds," Journal of Spacecraft and Rockets, Vol. 28, No. 1, Jan.-Feb. 1991, pp. 31-39.

4. Yoon, S., Jameson, A., and Kwak, D., "Effect of Artificial Diffusion Schemes on Multigrid Convergence," AIAA Paper 95-1670, June 1995.

5. Pulliam, T. and Venkataswaran, S., Private Communications. 